\title{
An Efficient Algorithm for Current Source Localization with Tetrodes
}

\author{
Chang Won Lee, Hieu Dang, and Zoran Nenadic
}

\begin{abstract}
In this article we develop a simple algorithm for localization of electrical sources in neurons. The algorithm is based on multiple measurements of neuron's action potentials acquired from multiple sensors, such as tetrodes. We show that in many cases this problem can be solved analytically. We also give an alternative formulation of the problem that is applicable when the analytical approach has no solution. We further show that the problem of source localization and the estimation of the source strength are coupled and can be solved simultaneously. Our method was tested on a singleneuron model with complex dendritic geometry and realistically modeled membrane dynamics. Simulation results suggest that our technique is capable of accurately estimating the location and strength of nearby current sources.
\end{abstract}

\section{INTRODUCTION}

Recent advances in the development of extracellular recording technology for the brain have been instrumental to address complex scientific questions such as neural population coding, somatosensory organization, and neural network connectivity. Recording is typically accomplished by placing multiple high-impedance electrodes (microelectrodes) [1], [2], or micro-machined silicon electrode arrays [3], [4], in a close proximity to active neurons and by measuring voltage perturbations caused by neurons' action potentials (APs). Similarly, microelectrodes with multiple closely spaced sensors have been shown to improve the signal-to-noise ratio (SNR), thereby facilitating the identification of a single neuron activity against the background noise and the activities of nearby neurons - a process commonly referred to as singleunit isolation [5].

Here, we report on a simple algorithm that extends the use of multi-sensor recording electrodes beyond a singleunit isolation. In particular, we show how a neural signal from an array of 4 sensors (a tetrode) can be used to estimate the location of a current source as well as the strength of the source. Our algorithm is based on a simple model of propagation of electric potentials in a volume conductor, referred here as forward model. The estimation of the source location and strength is then posed as an inverse problem. If the forward model provides a "good" fit to measured potentials, this inversion is exact and yields a closed form solution. In the presence of noise and distributed sources, which more closely correspond to reality, the exact inversion is not guaranteed and we pose the inversion as an

C.W. Lee is with the Department of Biomedical Engineering, University of California, Irvine, CA 92697, USA Cwlee@uci.edu

$\mathrm{H}$. Dang is with the Department of Biomedical Engineering, University of California, Irvine, CA 92697, USA hdang@uci.edu

Z. Nenadic is with the Departments of Biomedical Engineering and Electrical Engineering and Computer Science, University of California, Irvine, CA 92697, USA znenadic@uci.edu optimization problem by minimizing the mismatch between the measured potentials and the potentials predicted by the forward model.

\section{BACKGROUND AND SIGNIFICANCE}

Localization of neural sources is a problem common to various brain imaging modalities, such as electroencephalography (EEG), or magneto-encephalography (MEG). Despite the fact that EEG signals and extracellular potentials are closely related, the idea of source localization based on extracellular potentials appears to be largely unexploited. Oweiss and Anderson [6] proposed a source localization algorithm based on a planar sensor architecture, commonly referred to as the Michigan probe [7]. Their technique uses a forward model based on finite element analysis, which coupled with optimization yields an estimate of the source location. While details of their optimization approach were not disclosed in [6], the complexity of the forward model implies the use of search techniques. Chelaru and Jog [8] developed a source localization algorithm for the tetrode architecture based on a simple forward model, which is similar in spirit to the one proposed here. However, there are several important differences which will be pointed out in Section III. Recent studies [9], [10], report recordings from a large number of cortical units with high-density two-dimensional (2-D) silicon arrays. Signals from multiple sensors were then used to estimate the vertical positions of neurons or their 2-D projections. These approaches, however, have no basis in volume conductor theory or biophysics, and seem largely heuristic. In addition, no attempt was made to localize sources in 3-D.

Localization of neural signal sources is potentially beneficial to several research areas. For example, it could alleviate the guidance of microelectrodes in acute recording experiments, which are tedious and time consuming. Localization could also help develop better feedback control algorithms for stabilization of extracellular recordings [11], [12]. This could lead to improved experimental productivity and higher quality data. In chronic recording experiments, the estimation of the position and strength of signal sources will have important implications for long-term studies of neuronal populations, such as neuronal circuit connectivity [10], extracellular current source density [9], and functional organization of cell assemblies [13].

\section{SOURCE LOCALIZATION ALGORITHM}

When an AP is initiated, the electric current enters a neuron through its active membrane, flows through the cytoplasm, and then leaves the neuron through regions of passive 
membrane [14]. Therefore, when viewed from the outside, the neuron behaves as a continuum of current sources and sinks. For localization purposes, however, this model is overly complex; in a simplified scenario the neuron can be modeled as a single point source (or sink), located at position, $(x, y, z)$, with the time-varying current strength $I(t)$. Assuming extracellular space is an unbounded, isotropic, and homogeneous volume conductor with a conductivity (per unit length) $\sigma$, the potential at a sensor $S_{i}$ is found as

$$
\phi_{i}(t)=\frac{I(t)}{4 \pi \sigma r_{i}(x, y, z)}
$$

where $r_{i}(x, y, z)=\sqrt{\left(x-x_{i}\right)^{2}+\left(y-y_{i}\right)^{2}+\left(z-z_{i}\right)^{2}}$ and $\left(x_{i}, y_{i}, z_{i}\right)$ is the location of the sensor $S_{i}$. Note that $\phi_{i}(t)<0$ indicates the presence of a sink rather than a source. The time in (1) is fixed to $t=t^{*}$, where $t^{*}$ is chosen by the user (typically the time at which AP attains the peak value). For 4 sensors, $S_{i}(i=0,1,2,3)$, the forward model (1) can be rewritten as

$$
\left(x-x_{i}\right)^{2}+\left(y-y_{i}\right)^{2}+\left(z-z_{i}\right)^{2}=\frac{k^{2}}{\phi_{i}^{2}} \quad \forall i
$$

where $k=I\left(t^{*}\right) /(4 \pi \sigma)$ and $\phi_{i}=\phi_{i}\left(t^{*}\right)$. Note that (2) represents the equation of a sphere, centered at $S_{i}$, with the radius inversely proportional to the potential, $\phi_{i}$. The solution to (2) has a geometric interpretation: the source lies at the point of intersection of the 4 spheres. The localization scheme proposed in [8] uses essentially the same system of equations as (2), although the solution was found numerically. Note, however, that the nonlinearity in (2) is quadratic, hence the solution may be obtained in a closed form. Secondly, quadratic equations normally yield two solutions, and depending on initial conditions, numerical schemes may converge to the confounding solution. Finally, the system (2) may have no real solution, e.g. the 4 spheres may not have a common intersection point.

To solve (2) analytically, we define $\left(x_{0}, y_{0}, z_{0}\right)$ as the origin, yielding

$$
x^{2}+y^{2}+z^{2}=\frac{k^{2}}{\phi_{0}^{2}}
$$

By subtracting (2) with $i=1,2,3$, from (3) we obtain

$$
2 x x_{i}+2 y y_{i}+2 z z_{i}-R_{i}^{2}=\frac{k^{2}}{\phi_{0}^{2}-\phi_{i}^{2}} \quad i=1,2,3
$$

where $R_{i}^{2}=x_{i}^{2}+y_{i}^{2}+z_{i}^{2}$. Using vector notation, we have

$$
2\left[\begin{array}{lll}
x_{1} & y_{1} & z_{1} \\
x_{2} & y_{2} & z_{2} \\
x_{3} & y_{3} & z_{3}
\end{array}\right]\left[\begin{array}{l}
x \\
y \\
z
\end{array}\right]=k^{2}\left[\begin{array}{c}
\phi_{01} \\
\phi_{02} \\
\phi_{03}
\end{array}\right]+\left[\begin{array}{c}
R_{1}^{2} \\
R_{2}^{2} \\
R_{3}^{2}
\end{array}\right]
$$

where $\phi_{i j}=\frac{1}{\phi_{i}^{2}}-\frac{1}{\phi_{j}^{2}}$. Written in a compact form, this yields

$$
2 \mathbf{C X}=k^{2} \boldsymbol{\Phi}+\mathbf{R}
$$

with the obvious definitions of $\mathbf{C}, \mathbf{X}, \boldsymbol{\Phi}$, and $\mathbf{R}$. If the sensors are not confined to a plane, the matrix $\mathbf{C}$ is invertible and the solution to (5) is simply

$$
\mathbf{X}=\frac{1}{2} \mathbf{C}^{-1}\left(k^{2} \boldsymbol{\Phi}+\mathbf{R}\right)
$$

Note that for planar sensor arrays (e.g. the Michigan probe), C is not likely to be invertible. To complete the solution (6), the constant $k$ must be calculated. To this end, we substitute (6) into (3) $\left(x^{2}+y^{2}+z^{2}=\mathbf{X}^{T} \mathbf{X}\right)$ which yields

$$
\boldsymbol{\Phi}^{T} \mathbf{Q} \boldsymbol{\Phi} k^{4}+\left(2 \boldsymbol{\Phi}^{T} \mathbf{Q} \mathbf{R}-\frac{4}{\phi_{0}^{2}}\right) k^{2}+\mathbf{R}^{T} \mathbf{Q} \mathbf{R}=0
$$

where $\mathbf{Q}:=\left(\mathbf{C} \mathbf{C}^{T}\right)^{-1}$. Since (7) is a biquadratic equation in $k$, its closed form solution is easily found. Generally, (7) has 2 solutions, $k_{(1)}^{2}$ and $k_{(2)}^{2}$, which when substituted in (6) will yield 2 possible source locations. However, the spurious solution normally represents the reflection of the true solution through the surface of the tetrode and is easily identified. An important difference between our approach and the one proposed in [8] is that once $k^{2}$ is known from (7), we can estimate the current at the source as $I\left(t^{*}\right)=4 \pi \sigma k$. After eliminating the spurious solution, there will be 2 such solutions, but one of them can be eliminated based on the sign of $\phi_{i}$. Finally, note that for practical purposes, estimating $I\left(t^{*}\right)$ requires the knowledge of the extracellular conductivity, $\sigma$.

When the forward model (1) is exact, the solution (6) is guaranteed to exist in $\mathbb{R}^{3}$. However, if there is a mismatch between $\phi_{i}$, predicted by the model, and measured potentials, denoted by $\bar{\phi}_{i}$, the solution of (7), and consequently (6), may not be real. This is likely to be found in experimental situations for at least a couple of reasons: (i) the potentials are not generated by a single source, and (ii) the presence of noise, $\eta_{i}$, distorts the potentials (e.g. $\bar{\phi}_{i}=\phi_{i}+\eta_{i}$ ). Note that when the real solutions do not exist, numerical schemes will fail to converge, which is consistent with the study in [8], where failed convergence was reported in about $13 \%$ of cases. To circumvent this situation, we propose to reformulate the source localization problem within an optimization framework. We define a cost function as a mismatch between the measured potential, $\bar{\phi}_{i}$, and model's prediction, $\phi_{i}$,

$$
\mathcal{J}(k, x, y, z)=\frac{1}{2} \sum_{i=0}^{3}\left[\bar{\phi}_{i}-\frac{k}{r_{i}(x, y, z)}\right]^{2}
$$

and we proceed by finding its minimum. Note that $\mathcal{J} \geq 0$ and that the equality holds if and only if $\bar{\phi}_{i}=\phi_{i}$, in which case the minimizer of (8) can be found analytically from (6) and (7). In general, however, the solution cannot be found analytically, and we resort to numerical techniques. Both the gradient and the Hessian of $\mathcal{J}$ can be calculated in a closed form, which makes the minimization of $\mathcal{J}$ amenable to (fast) Newton's method. In summary, our source localization algorithm first attempts to find the closed form solution based on (6) and (7). If complex conjugate solutions appear, their real parts are taken as an initial condition for the minimization of (8), and the minimizer of $\mathcal{J}$ is taken as a solution. 

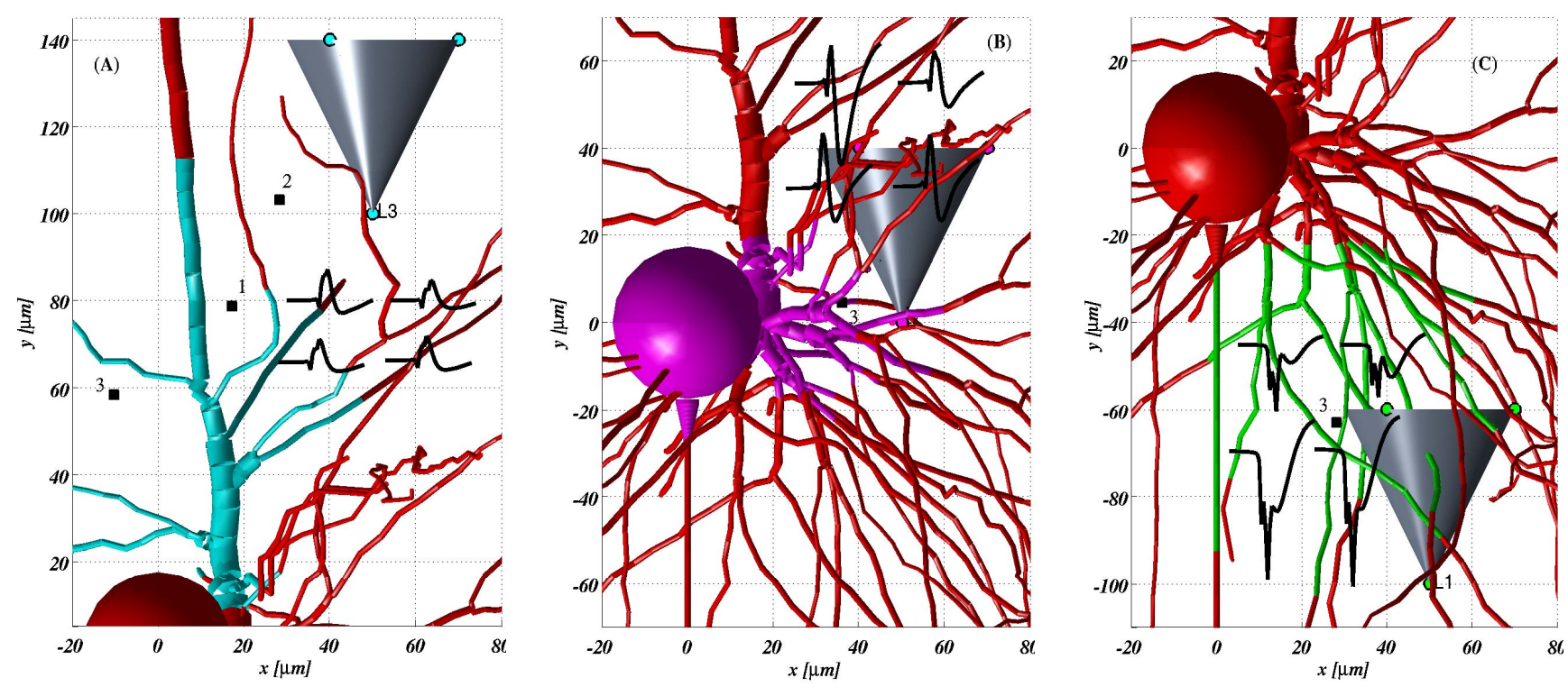

Fig. 1. (A) Tetrode in the region of the proximal basal dendrite. (B) Tetrode in the vicinity of the soma. (C) Tetrode in the region of the distal basal dendrite. The straight line represents the axon, the cone-like cylinder is the axon hillock, and the adjacent axonal region is the initial segment. The tetrode sensors are shown as circles (4th sensor not visible). The estimated source locations are shown as black squares ( 3 based on $t^{*}$ corresponding to the peak of APs, 2 and 1 based on $t^{*}$ that precedes the peak by 1 and 2 samples, respectively). APs recorded by the 4 sensors are shown as black traces (duration $2 m s)$.

\section{EXPERIMENTAL RESULTS}

When tested with a single current source (sink), located at an arbitrary position, our algorithm gave a 100\% accuracy in terms of both the location and strength of the source. This is not surprising since in this case the forward model (1) is correct, and its inversion reduces to solving (6) and (7). To make the problem more realistic, we used a computational model of a pyramidal neuron from layer 5 of the cat visual cortex [15]. Fig. 1 illustrates the complex dendritic geometry of this type of neuron. Low-density sodium channels were present in the soma and dendrites, while the axon hillock (AH) and initial segment (IS) had high-density sodium channels. Fast potassium channels were present in the axon and soma, but were not present in the dendrites. This type of channel distribution is responsible for spike initiation at the axon initial segment [15]. The neuron was activated by synapses uniformly distributed throughout the dendrites [16]. Intracellular potentials were computed using the NEURON simulator [17], with 3720 isopotential segments, while extracellular potentials were simulated using the line source approximation [16], [12]. The sampling rate was kept at $20 \mathrm{kHz}$ throughout simulations. The tetrode geometry specifications were adopted from a commercial supplier [18], and the distance between sensors was between 20 and $40 \mu \mathrm{m}$.

Based on the channel distribution, the strongest transmembrane currents are found in the AH/IS area, although the soma is a significant contributor of the potential due to its large surface area. The contribution of the dendrites to the potential is mostly local, and falls off rapidly with distance. Thus, it is expected that the estimated source location will be somewhere in the vicinity of the soma-AH-IS complex.
When the tetrode is relatively distant from the cell, the principal sources (soma, AH, IS) appear localized, and the estimated source location does fall in the vicinity of the soma. The contribution of the dendrites is negligible in this case. At the same time, the localization invariably admits a closed form solution, based on (6) and (7). This is hardly surprising given the fact that the forward model (1) provides a good approximation of highly localized sources. As the tetrode moves closer to the soma (Fig. 1 (A)), the principal current sources appear more diffused and the localization based on the inversion of (1) becomes less accurate, although the closed form solution is still feasible. Interestingly, the spurious solutions tend to be located inside, or near the surface of the tetrode, and were easy to rule out. If the tetrode is moved forward into the dendritic tree (Fig. 1 (B)(C)), some of the dendrites wind up very close to the surface of the tetrode, and their effect is no longer negligible. These local contributions may add to the variability of recorded APs. In these situations, the inverse problem rarely admits a closed form solution, and the optimization must be performed instead. Furthermore, the effect of local dendrites seem to bias the estimated source locations toward the tetrode (Fig. 1 (B)(C)).

Since localization is based on a specific time $t^{*}$, we investigated how varying $t^{*}$ affects the estimates (Fig. 1 (A)). When the peak of APs is used (marked by 3), the estimated source location is closer to the soma, albeit biased by the large dendritic trunk that passes nearby. Moving $t^{*}$ one sample before the peak of APs, causes the estimate (marked by 2 ) to move toward the tetrode. Moving $t^{*}$ forward by two samples causes similar effect (position marked by 1). Thus, we conclude that the estimated sources tend to shift dynam- 
ically, as different phases of APs are used for localization. This phenomenon is explained as follows: the peak of APs are mostly due to the principal sources (soma, AH, IS). Due to relatively sharp rising edge of APs, moving $t^{*}$ forward by a sample, or a couple of samples, changes the measured potentials, $\bar{\phi}_{i}$, significantly. At this point the contribution of the principal sources has not been fully developed and is more comparable to that of the dendrites, hence the shift. For experimental data, however, any feature of APs other than its peak value is likely to be very sensitive to noise. Thus, in experimental set-up, we expect the peak of APs to be used for localization purposes, as was done in [8].

We now turn to the estimation of the source strength, $I\left(t^{*}\right)$. If the source (sink) was confined to a single segment, it would be expected that $I\left(t^{*}\right)$ approximates the transmembrane current of the segment at time $t^{*}$. Since a number of segments are involved in the generation of the potential, $I\left(t^{*}\right)$ is likely to represent the gross strength of the most relevant segments. To test this hypothesis, the segments were ranked according to their distance to the estimated source location, and the transmembrane currents of the $n$ closest segments were added. The number of segments, $n$, was increased until the gross current matched the estimated source strength [ $n=120$ in Fig. 1 (A)(C), $n=295$ in Fig. 1 (B)]. For convenience, these relevant segments are shown in cyan, magenta and green, for the 3 respective tetrode locations. While largely heuristic, this approach essentially agrees with our prior conclusions: the dendritic trunk seems very relevant in Fig. 1 (A), the soma and AH are relevant in Fig. 1 (B), while IS is relevant in Fig. 1 (C). Also note that in the latter 2 cases, the relevant segments involve a large number of dendrites.

\section{CONClusion And Future Work}

Based on elementary ideas from volume conductor theory, we have developed a computationally simple neural source localization algorithm for a tetrode sensor layout. We have shown that the problems of source localization and source current estimation can be solved simultaneously, provided that the conductivity of extracellular medium is known. When the sources of neural signal are localized in space, this inverse problem can be solved analytically. Otherwise, a simple optimization routine, based on Newton's method, is used.

To investigate the applicability of our algorithm in experimental set-up, the present work needs extensions in two areas: (i) the addition of realistically modeled neural noise to mimic noisy recording conditions, (ii) the modification of the forward model (1) to a more realistic model, such as a dipole (source + sink). Both extensions are non-trivial. We have recently pointed out the importance and implications of realistically modeled neural noise [19], [20] in the context of neural signal processing. Noise acquired during electrophysiologic recordings has been instrumental in uncovering some limitations of classical approaches to noise modeling. Dipole localization is an inherently ill-posed problem, and its solution will likely require some form of regularization [21].
However, similar problems are routinely faced in EEG dipole localization, where they have been handled successfully.

\section{REFERENCES}

[1] V. B. Mountcastle and H. J. Reitboeck and G. F. Poggio and M. A. Steinmetz. Adaptation of the reitboeck method of multiple microelectrode recording to the neocortex of the waking monkey. $J$. Neurosci. Meth., 36:77-84, 1991.

[2] R. Eckhorn and U. Thomas. A new method for the insertion of multiple microprobes into neural and muscular tissue, including fiber electrodes, fine wires, needles and microsensors. J. Neurosci. Meth., 1993.

[3] K. D. Wise. Micromachined interfaces to the cellular world. Sensor. Mater, 10(6):385-395, 1998.

[4] P. Norlin, M. Kindlundh, A. Mouroux, K. Yoshida, and U. G. Hofmann. A 32-site neural recording probe fabricated by DRIE of SOI substrates. J. Micromech. Microeng., 14(4):414-419, 2002.

[5] C. M. Gray, P. E. Maldonado, M. Wilson, and B. McNaughton. Tetrodes markedly improve the reliability and yield of multiple singleunit isolation from multi-unit recordings in cat striate cortex. $J$. Neurosci. Meth., 63(1-2):43-54, 1995.

[6] K.G. Oweiss and D.J. Anderson. Neural source localization using advanced sensor array signal processing techniques. In in Proc. of the 23rd Annual International Conference of the IEEE Engineering in Medicine and Biology Society, pages 707-710, 2001.

[7] K.L. Drake and K.D. Wise and J. Farraye and D. J. Anderson and S.L. BeMent. Performance of planar multisite microprobes in recording extracellular single-unit intracortical activity. IEEE T. Bio-med. Eng., 35:719-732, 1988.

[8] M.I. Chelaru and M.S. Jog. Spike source localization with tetrodes. J. Neurosci. Meth., 142(2):305-315, 2005.

[9] J. Csicsvari, D. A. Henze, B. Jamieson, K. D. Harris, A. Sirota, P. Bartho, K. D. Wise, and G. Buzsaki. Massively parallel recording of unit and local field potentials with silicon-based electrodes. J. Neurophysiol., 90(2):1314-1323, 2003.

[10] P. Bartho, H. Hirase, L. Monconduit, M. Zugaro, K. D. Harris, and G. Buzsaki. Characterization of neocortical principal cells and interneurons by network interactions and extracellular features. $J$. Neurophysiol., 92(1):600-608, 2004.

[11] J. G. Cham, E. A. Branchaud, Z. Nenadic, B. Greger, R. A. Andersen, and J. W. Burdick. Semi-chronic motorized microdrive and control algorithm for autonomously isolating and maintaining optimal extracellular action potentials. J. Neurophysiol., 93(1):570-579, 2005.

[12] Z. Nenadic and J. W. Burdick. A control algorithm for autonomous optimization of extracellular recordings. IEEE T. Bio-med. Eng., 53(5):941-955, 2006.

[13] K. D. Harris, J. Csicsvari, H. Hirase, G. Dragoi, and G. Buzsaki. Organization of cell assemblies in the hippocampus. Nature, 424(6948):552-556, 2003.

[14] D.R. Humphrey. Electrophysiological Techniques. Society for Neuroscience, Atlanta, 1979.

[15] Z.F. Mainen and T.J. Sejnowski. Influence of dendritic structure on firing pattern in model neocortical neurons. Nature, 382:363-366, 1996.

[16] G. R. Holt and C. Koch. Electrical interactions via the extracellular potential near cell bodies. J. Comp. Neurosci., 6:169-184, 1999.

[17] M.L. Hines and N.T. Carnevale. The neuron simulation environment. Neural Comput., 9:1179-1209, 1997.

[18] Tetrode datasheet. http://www. thomasrecording.com.

[19] Zoran Nenadic and Joel W. Burdick. Spike detection using the continuous wavelet transform. IEEE T. Bio-med. Eng., 52(1):74-87, 2005.

[20] R. Benitez and Z. Nenadic. Robust unsupervised detection of action potentials with probabilistic models. IEEE T. Bio-med Eng. (in revision).

[21] A.N. Tikhonov and V.Y. Arsenin. Solutions to Ill-Posed Problems. Winston, Washington, DC, 1977. 